www.asianjournalofmycology.org Article

Doi 10.5943/ajom/2/1/16

\title{
Arthrinium setostromum (Apiosporaceae, Xylariales), a novel species associated with dead bamboo from Yunnan, China
}

\author{
Jiang $\mathrm{HB}^{1,2,3}$, Hyde $\mathrm{KD}^{1,2}$, Doilom $\mathrm{M}^{1,2,4}$, Karunarathna $\mathrm{SC}^{2,4,5}$, Xu $\mathrm{JC}^{2,4}$ and \\ Phookamsak $R^{1,2,4^{*}}$
}

${ }^{1}$ Center of Excellence in Fungal Research, Mae Fah Luang University, Chiang Rai 57100, Thailand

${ }^{2}$ Key Laboratory for Economic Plants and Biotechnology, Kunming Institute of Botany, Chinese Academy of Sciences, Kunming 650201, Yunnan, China

${ }^{3}$ School of Science, Mae Fah Luang University, Chiang Rai 57100, Thailand

${ }^{4}$ East and Central Asia Regional Office, World Agroforestry Centre (ICRAF), Kunming 650201, Yunnan, China

${ }^{5}$ Department of Biology, Faculty of Science, Chiang Mai University, Chiang Mai 50200, Thailand

Jiang HB, Hyde KD, Doilom M, Karunarathna SC, Xu JC, Phookamsak R 2019 - Arthrinium setostromum (Apiosporaceae, Xylariales), a novel species associated with dead bamboo from Yunnan, China. Asian Journal of Mycology 2(1), 254-268, Doi 10.5943/ajom/2/1/16

\begin{abstract}
Arthrinium setostromum sp. nov., collected from dead branches of bamboo in Yunnan Province of China, is described and illustrated with the sexual and asexual connections. The sexual morph of the new taxon is characterized by raised, dark brown to black, setose, lenticular, 1-3loculate ascostromata, immersed in a clypeus, unitunicate, 8-spored, broadly clavate to cylindricclavate asci and hyaline apiospores, surrounded by an indistinct mucilaginous sheath. The asexual morph develops holoblastic, monoblastic conidiogenesis with globose to subglobose, dark brown, 0-1-septate conidia. Arthrinium setostromum can be distinguished from other phylogenetically closely related species by its setose ascostroma, with setae raising through a split of the clypeus. Maximum likelihood, maximum parsimony and bayesian inference analyses based on a catenated ITS, LSU, TEF1- $\alpha$ and TUB2 DNA sequence dataset demonstrate that $A$. setostromum is a distinct new species phylogenetically closely related to A. garethjonesii, and clusters with A. bambusae, A. mytilomorphum and A. subroseum.
\end{abstract}

Key words - 1 new species - bambusicolous fungi - holomorph - multi-gene phylogeny Sordariomycetes

\section{Introduction}

Arthrinium was introduced by Kunze (1817) with A. caricicola Kunze \& J.C. Schmidt as the type species. Traditionally, Arthrinium (as an asexual morph) was linked to Apiospora Sacc. (as a sexual morph) (Ellis 1971, Seifert et al. 2011). Pteroconium Sacc. ex Grove was also reported as an asexual morph of Apiospora, however, the genus has a large morphological difference from Arthrinium in conidial shape (Ellis 1971, Wang et al. 2018). Crous \& Groenewald (2013) treated Pteroconium and Cordella Speg. as the synonym of Arthrinium based on molecular phylogenetic data. In general, Apiospora was regarded as a synonym of Arthrinium based on the one fungus-one name policy (Hawksworth et al. 2011, Crous \& Groenewald 2013).

The sexual morph of Arthrinium is characterized by immersed to erumpent through a longitudinal split, uni- to multi-loculate, dark brown to black, lenticular, or dome-shaped 
ascostromata with or without setae growing from perithecia, 8-spored, unitunicate, broadly clavate to cylindric-clavate asci and hyaline, ellipsoidal, inequilaterally, 2-3-seriate, 1-septate near the lower end ascospores with or without sheath (Senanayake et al. 2015, Dai et al. 2016, 2017, Maharachchikumbura et al. 2016, Pintos et al. 2019). The asexual morphs of Arthrinium includes coelomycetous and hyphomycetous forms; for example, A. hyphopodii D.Q. Dai \& K.D. Hyde and A. qinlingense C.M. Tian \& N. Jiang produced coelomycetous asexual morph (Senanayake et al. 2015, Dai et al. 2016, Jiang et al. 2018), while A. esporlense Pintos \& P. Alvarado formed hyphomycetous asexual morph (Pintos et al. 2019).

The hyphomycetous asexual morph of Arthrinium is described as subhyaline or pale brown, subcylindrical, septate conidiophores reduced to conidiogenous cells or arising from basal cells, forming conidia laterally or terminally, with or without setae, hyaline or subhyaline to pale brown, doliiform to ampulliform or subcylindrical, smooth or finely roughened, holoblastic, monoblastic or polyblastic conidiogenous cells and 0-1-septate, dark brown or brown to pale olivaceous conidia, vary in shape (e.g. lenticular, globose to subglobose, elongated to ellipsoidal, fusiform, spindleshaped, lemon-shaped, etc.), with or without a truncate base and a germ-slit (Singh et al. 2012, Crous \& Groenewald 2013, Crous et al. 2015, Hyde et al. 2016, Dai et al. 2017, Wang et al. 2018, Pintos et al. 2019, Yan et al. 2019).

The coelomycetous asexual morph of Arthrinium is characterized by immersed in host tissue or superficial on media, black, globose to subglobose, coriaceous conidiomata, forming subhyaline or pale brown, hyphoid, cylindrical, septate conidiophores reduced to conidiogenous cells or arising from basal cells, subhyaline or pale brown, cylindrical, smooth or with verrucose, holoblastic, monoblastic or polyblastic conidiogenous cells and dark brown, globose to subglobose, smooth, conidia with or without a truncate basal scar and a germ-slit (Senanayake et al. 2015, Dai et al. 2017, Jiang et al. 2018, Yang et al. 2019).

Arthrinium species are widely distributed (Sharma et al. 2014). Species of the genus can be endophytes, pathogens or saprobes, usually isolated from soil debris, plants, lichens and marine algae (Senanayake et al. 2015, Wijayawardene et al. 2017), as well as from gut of insect (Crous et al. 2015) and erythematous nodules of human (Sharma et al. 2014).

In this study, we introduce a novel member of Arthrinium with the sexual and asexual morph linkage, collected from Yunnan, China, based on distinct morphological characteristics and multigene phylogenetic analyses. The new taxon is illustrated and compared with similar taxa.

\section{Materials \& methods}

\section{Collection and examination of specimen, fungal isolation and conservation}

The fresh sample, collected from Mengla County, Xishuangbanna Dai Autonomous Prefecture of Yunnan Province, China was brought to the laboratory for observation and examination. Ascostromata appeared on the host surface were examined and captured using an Olympus stereo microscope series MoDELSZ2-ILST. Micromorphological features were examined and captured by a Nikon ECLIPSE Ni-U compound microscope connected with a Canon EOS $600 \mathrm{D}$ digital camera. The presence of mucilaginous sheath surrounded ascospores was detected by immersing in the Indian ink. The asci were stained by Melzer's reagent for checking the J-/J+ apical ring. Measurements of morphological structures (e.g. asci, ascospores, paraphyses, peridium, ascostromata, conidia, conidiogenous cells and conidiophores) were made in Tarosoft ${ }^{\circledR}$ Image Frame Work software version 0.9.7. Photographic plates were edited and combined in Adobe Photoshop CS6 software (Adobe Systems Inc., United States).

Single spore isolation was performed following the method described by Dai et al. (2017). Germinating ascospores were aseptically transferred to the medium plate of potato dextrose agar (PDA) for incubation under normal light at $20-25^{\circ} \mathrm{C}$. Fungal colonies were observed and morphology and size of colonies were recorded at weekly intervals for two months. After two months, the sporulation of the asexual morph of the new taxon was observed and examined by using the same method of the sexual morph examination. 
Herbarium materials are deposited in the herbarium of the Cryptogams Kunming Institute of Botany Academia Sinica (KUN-HKAS) and duplicated in the herbarium of Mae Fah Luang University, Chiang Rai, Thailand (MFLU). The ex-type living culture was conserved at the Kunming Institute of Botany Culture Collection (KUMCC) and Mae Fah Luang University Culture Collection (MFLUCC). Facesoffungi and Index Fungorum numbers are provided for the newly described taxon (Jayasiri et al. 2015, Index Fungorum 2019). The novel taxon was justified based on the guideline of Jeewon \& Hyde (2016).

\section{DNA extraction, PCR amplification and sequencing}

Total genomic DNA was extracted from fresh mycelia grown on PDA media with the Biospin Fungus Genomic DNA Extraction Kit (BioFlux ${ }^{\circledR}$, Hangzhou, and P.R. China) following the manufacturer's instructions.

Partial DNA sequences of the internal transcribed spacers (ITS1-5.8S-ITS2), the 28S large subunit rDNA (LSU) and the translation elongation factor 1-alpha (TEF1- $\alpha$ ), were amplified by primers ITS5 and ITS4 (White et al. 1990), LR0R and LR5 (Vilgalys \& Hester 1990) and EF1728F and EF-2 (O’Donnell et al. 1998, Carbone \& Kohn 1999), respectively.

PCR was performed in a $25 \mu \mathrm{l}$ total volume containing $2 \mu \mathrm{l}$ of DNA template, $1 \mu \mathrm{l}$ of each forward and reverse primers, $12.5 \mu \mathrm{l}$ of $2 \times$ Power Taq PCR Master Mix (mixture of EasyTaqTM DNA Polymerase, dNTPs, and optimized buffer, Beijing Bio Teke Corporation (Bio Teke), P.R. China) and $8.5 \mu$ of $\mathrm{ddH}_{2} \mathrm{O}$ (double-distilled water). The annealing temperatures were adjusted to $52^{\circ} \mathrm{C}$ for ITS and LSU, and $55^{\circ} \mathrm{C}$ for TEF1- $\alpha$. PCR products were sent to sequence at Sangon Biotech (Shanghai) Co., Ltd, China and TsingKe Biological Technology (Beijing) Co., Ltd, China.

\section{Phylogenetic analyses}

Through the BLASTn search tool in NCBI (https://blast.ncbi.nlm.nih.gov/Blast.cgi) based on newly generating ITS sequence data, we found out that our taxon related to Arthrinium (Apiosporaceae) and the closest taxon is A. garethjonesii D.Q. Dai \& H.B. Jiang (HKAS 96289) with $96.55 \%$ similarity. Further sequence matrices of ITS, LSU, TEF1- $\alpha$ and TUB2 were used to resolve the accurate phylogenetic position of our taxon based on the related sequences of taxa in Arthrinium obtained from GenBank (Table 1). Individual gene matrix was aligned with the online version of MAFFT v. 7.427 (Katoh et al. 2017) and improved manually in BioEdit v. 5.0.6 (Hall 2001). Individual gene alignments were analyzed separately by Randomized Axelerated Maximum Likelihood (RAxML) implemented in RAxMLGUI v. 1.0 (Stamatakis 2006, Silvestro \& Michalak 2011) for prior comparing of tree topologies. Further, a concatenated ITS, LSU, TEF1- $\alpha$ and TUB2 alignment was analyzed based on maximum likelihood (ML), maximum parsimony (MP) and Bayesian inference (BI) criteria.

ML analysis was carried out by RAxML implemented in RAxMLGUI v. 1.0 (Stamatakis 2006, Silvestro \& Michalak 2011). BI analysis was performed by MrBayes v. 3.2.1 (Ronquist et al. 2012). Bayesian posterior probabilities (BYPP) (Rannala \& Yang 1996, Zhaxybayeva \& Gogarten 2002) were evaluated based on Markov Chain Monte Carlo sampling (MCMC). Six simultaneous Markov chains were set up at 3,000,000 generations and trees were sampled every $100^{\text {th }}$ generation (yielded 30,000 total trees). The first 20\% trees, which represented the burn-in phase of the analysis, were discarded and the remaining trees were used for calculating PP in the $50 \%$ majority rule consensus tree. MP analysis was operated in PAUP v. 4.0b10 (Swofford 2002) based on the heuristic search option with 1,000 random stepwise addition replicates and the tree bisectionreconnection (TBR) as the branch-swapping algorithm. All informative characters were unordered and of equal weight. MaxTrees reset to 1,000 and gaps were treated as missing data. All parsimonious trees were saved. The tree length (TL), consistency index (CI), retention index (RI), rescaled consistency index (RC) and homoplasy index (HI) were calculated. Statistical supports for branches of the most parsimonious tree were estimated using maximum parsimony bootstrap (BS) analysis with 1,000 bootstrap replicates (Felsenstein 1985). 
Phylogenetic trees were represented by FigTree v. 1.4.0 (Rambaut 2012) and edited in Microsoft Office PowerPoint 2016 (Microsoft Inc., United States). Newly generated sequences in this study were deposited in GenBank (Table 1) and the final matrices used for the phylogenetic analyses were submitted to TreeBASE (www.treebase.org; accession number: 25246).

Table 1 List of fungal taxa and their GenBank accession numbers of the sequences used in this study.

\begin{tabular}{|c|c|c|c|c|c|}
\hline \multirow{2}{*}{ Species } & \multirow{2}{*}{ Strain no. } & \multicolumn{4}{|c|}{ GenBank Accession numbers } \\
\hline & & ITS & LSU & TEF1- $\alpha$ & TUB2 \\
\hline Arthrinium arundinis & CBS 106.12 & KF144883 & KF144927 & KF145015 & KF144973 \\
\hline Arthrinium arundinis & CBS 114316 & KF144884 & KF144928 & KF145016 & KF144974 \\
\hline Arthrinium arundinis & CBS 124788 & KF144885 & KF144929 & KF145017 & KF144975 \\
\hline Arthrinium arundinis & CBS 133509 & KF144886 & KF144930 & KF145018 & KF144976 \\
\hline Arthrinium arundinis & CBS 449.92 & KF144887 & KF144931 & KF145019 & KF144977 \\
\hline Arthrinium aureum & CBS 244.83 & AB220251 & KF144935 & KF145023 & KF144981 \\
\hline Arthrinium balearicum & CBS 145129 & MK014869 & MK014836 & MK017946 & MK017975 \\
\hline Arthrinium bambusae & LC7106 & KY494718 & KY494794 & KY806204 & KY705186 \\
\hline Arthrinium bambusae & LC7124 & KY494727 & KY494803 & KY806206 & KY705195 \\
\hline Arthrinium bambusae & LC7125 & KY494728 & KY494804 & KY705124 & KY705196 \\
\hline Arthrinium camelliae-sinensis & LC5007 & KY494704 & KY494780 & KY705103 & KY705173 \\
\hline Arthrinium camelliae-sinensis & LC8181 & KY494761 & KY494837 & KY705157 & KY705229 \\
\hline Arthrinium caricicola & CBS 145127 & MK014871 & MK014838 & MK017948 & MK017977 \\
\hline Arthrinium descalsii & CBS 145130 & MK014870 & MK014837 & MK017947 & MK017976 \\
\hline Arthrinium dichotomanthi & LC4950 & KY494697 & KY494773 & KY705096 & KY705167 \\
\hline Arthrinium dichotomanthi & LC8175 & KY494755 & KY494831 & KY705151 & KY705223 \\
\hline Arthrinium esporlense & CBS 145136 & MK014878 & MK014845 & MK017954 & MK017983 \\
\hline Arthrinium euphorbiae & IMI 285638b & AB220241 & AB220335 & 1 & AB220288 \\
\hline Arthrinium gaoyouense & CFCC 52301 & MH197124 & / & MH236793 & MH236789 \\
\hline Arthrinium gaoyouense & CFCC 52302 & MH197125 & l & MH236794 & MH236790 \\
\hline Arthrinium garethjonesii & KUMCC 16-0202 & KY356086 & KY356091 & / & / \\
\hline Arthrinium guizhouense & LC5318 & KY494708 & KY494784 & KY705107 & KY705177 \\
\hline Arthrinium guizhouense & LC5322 & KY494709 & KY494785 & KY705108 & KY705178 \\
\hline Arthrinium gutiae & CBS 135835 & KR011352 & KR149063 & KR011351 & KR011350 \\
\hline Arthrinium hispanicum & IMI 326877 & AB220242 & AB220336 & / & AB220289 \\
\hline Arthrinium hydei & CBS 114990 & KF144890 & KF144936 & KF145024 & KF144982 \\
\hline Arthrinium hydei & KUMCC 16-0204 & KY356087 & KY356092 & / & / \\
\hline Arthrinium hyphopodii & $\begin{array}{l}\text { MFLUCC 15- } \\
0003\end{array}$ & KR069110 & / & I & l \\
\hline Arthrinium hyphopodii & KUMCC 16-0201 & KY356088 & KY356093 & / & / \\
\hline Arthrinium hysterinum & ICMP 6889 & MK014874 & MK014841 & MK017951 & MK017980 \\
\hline Arthrinium ibericum & CBS 145137 & MK014879 & MK014846 & MK017955 & MK017984 \\
\hline Arthrinium italicum & CBS 145138 & MK014880 & MK014847 & MK017956 & MK017985 \\
\hline Arthrinium japonicum & IFO 30500 & AB220262 & AB220356 & / & AB220309 \\
\hline Arthrinium japonicum & IFO 31098 & AB220264 & AB220358 & l & AB220311 \\
\hline Arthrinium jatrophae & AMH-9556 & HE981191 & l & l & / \\
\hline Arthrinium jatrophae & AMH-9557 & JQ246355 & l & I & I \\
\hline
\end{tabular}


Table 1 Continued.

\begin{tabular}{|c|c|c|c|c|c|}
\hline \multirow{2}{*}{ Species } & \multirow{2}{*}{ Strain no. } & \multicolumn{4}{|c|}{ GenBank Accession numbers } \\
\hline & & ITS & LSU & TEF1- $\alpha$ & TUB2 \\
\hline Arthrinium jiangxiense & LC4494 & KY494690 & KY494766 & KY705089 & KY705160 \\
\hline Arthrinium jiangxiense & LC4577 & KY494693 & KY494769 & KY705092 & KY705163 \\
\hline Arthrinium kogelbergense & CBS 113332 & KF144891 & KF144937 & KF145025 & KF144983 \\
\hline Arthrinium kogelbergense & CBS 113333 & KF144892 & KF144938 & KF145026 & KF144984 \\
\hline Arthrinium kogelbergense & CBS 113335 & KF144893 & KF144939 & KF145027 & KF144985 \\
\hline Arthrinium kogelbergense & CBS 117206 & KF144895 & KF144941 & KF145029 & KF144987 \\
\hline Arthrinium locuta-pollinis & CGMCC 3.18782 & MF939595 & / & MF939616 & MF939622 \\
\hline Arthrinium longistromum & MFLUCC 11-0479 & KU940142 & KU863130 & 1 & l \\
\hline Arthrinium longistromum & MFLUCC 11-0481 & KU940141 & KU863129 & l & / \\
\hline Arthrinium malaysianum & CBS 102053 & KF144896 & KF144942 & KF145030 & KF144988 \\
\hline Arthrinium marii & CBS 497.90 & AB220252 & KF144947 & KF145035 & KF144993 \\
\hline Arthrinium mediterranei & IMI 326875 & AB220243 & AB220337 & l & AB220290 \\
\hline Arthrinium minus & CBS 145131 & MK014872 & MK014839 & MK017949 & MK017978 \\
\hline Arthrinium mytilomorphum & DAOM 214595 & KY494685 & / & / & / \\
\hline Arthrinium neosubglobosa & JHB006 & KY356089 & KY356094 & l & l \\
\hline Arthrinium neosubglobosa & KUMCC 16-0203 & KY356090 & KY356095 & / & / \\
\hline Arthrinium obovatum & LC4940 & KY494696 & KY494772 & KY705095 & KY705166 \\
\hline Arthrinium obovatum & LC8177 & KY494757 & KY494833 & KY705153 & KY705225 \\
\hline Arthrinium obovatum & LC8178 & KY494758 & KY494834 & KY705154 & KY705226 \\
\hline Arthrinium ovatum & CBS 115042 & KF144903 & KF144950 & KF145037 & KF144995 \\
\hline $\begin{array}{l}\text { Arthrinium } \\
\text { paraphaeospermum }\end{array}$ & MFLUCC 13-0644 & KX822128 & KX822124 & / & / \\
\hline Arthrinium phaeospermum & CBS 114314 & KF144904 & KF144951 & KF145038 & KF144996 \\
\hline Arthrinium phaeospermum & CBS 114315 & KF144905 & KF144952 & KF145039 & KF144997 \\
\hline Arthrinium phaeospermum & CBS 114317 & KF144906 & KF144953 & KF145040 & KF144998 \\
\hline Arthrinium phaeospermum & CBS 114318 & KF144907 & KF144954 & KF145041 & KF144999 \\
\hline Arthrinium phragmites & СРC 18900 & KF144909 & KF144956 & KF145043 & KF145001 \\
\hline Arthrinium phyllostachium & MFLUCC 18-1101 & MK351842 & МH368077 & MK340918 & MK291949 \\
\hline Arthrinium piptatheri & CBS 145149 & МК014893 & MK014860 & MK017969 & / \\
\hline $\begin{array}{l}\text { Arthrinium } \\
\text { pseudoparenchymaticum }\end{array}$ & LC7234 & KY494743 & KY494819 & KY705139 & KY705211 \\
\hline $\begin{array}{l}\text { Arthrinium } \\
\text { pseudoparenchymaticum }\end{array}$ & LC8173 & KY494753 & KY494829 & KY705149 & KY705221 \\
\hline $\begin{array}{l}\text { Arthrinium } \\
\text { pseudoparenchymaticum }\end{array}$ & LC8174 & KY494754 & KY494830 & KY705150 & KY705222 \\
\hline Arthrinium pseudosinense & CPC 21546 & KF144910 & KF144957 & KF145044 & / \\
\hline Arthrinium pseudospegazzinii & CBS 102052 & KF144911 & KF144958 & KF145045 & KF145002 \\
\hline Arthrinium pterospermum & CBS 123185 & KF144912 & KF144959 & / & KF145003 \\
\hline Arthrinium pterospermum & СРC 20193 & KF144913 & KF144960 & KF145046 & KF145004 \\
\hline Arthrinium puccinioides & CBS 549.86 & AB220253 & AB220347 & / & AB220300 \\
\hline Arthrinium qinlingense & CFCC 52303 & MH197120 & / & MH236795 & MH236791 \\
\hline Arthrinium qinlingense & CFCC 52304 & MH197121 & / & MH236796 & MH236792 \\
\hline Arthrinium rasikravindrii & MFLUCC 11-0616 & KU940144 & KU863132 & / & I \\
\hline
\end{tabular}


Table 1 Continued.

\begin{tabular}{|c|c|c|c|c|c|}
\hline \multirow{2}{*}{ Species } & \multirow{2}{*}{ Strain no. } & \multicolumn{4}{|c|}{ GenBank Accession numbers } \\
\hline & & ITS & LSU & TEF1- $\alpha$ & TUB2 \\
\hline Arthrinium rasikravindrii & $\begin{array}{l}\text { MFLUCC 15- } \\
0203\end{array}$ & KU940143 & KU863131 & / & / \\
\hline Arthrinium rasikravindrii & NFCCI 2144 & JF326454 & / & / & / \\
\hline Arthrinium sacchari & CBS 212.30 & KF144916 & KF144962 & KF145047 & KF145005 \\
\hline Arthrinium sacchari & CBS 301.49 & KF144917 & KF144963 & KF145048 & KF145006 \\
\hline Arthrinium sacchari & CBS 372.67 & KF144918 & KF144964 & KF145049 & KF145007 \\
\hline Arthrinium sacchari & CBS 664.74 & KF144919 & KF144965 & KF145050 & KF145008 \\
\hline Arthrinium saccharicola & CBS 191.73 & KF144920 & KF144966 & KF145051 & KF145009 \\
\hline Arthrinium saccharicola & CBS 463.83 & KF144921 & KF144968 & KF145053 & KF145011 \\
\hline Arthrinium saccharicola & CBS 831.71 & KF144922 & KF144969 & KF145054 & KF145012 \\
\hline Arthrinium serenense & IMI 326869 & AB220250 & AB220344 & / & AB220297 \\
\hline Arthrinium setostromum & $\begin{array}{l}\text { KUMCC 19- } \\
0217\end{array}$ & MN528012 & MN528011 & MN527357 & / \\
\hline Arthrinium sporophleum & CBS 145154 & MK014898 & MK014865 & MK017973 & MK018001 \\
\hline Arthrinium subglobosum & $\begin{array}{l}\text { MFLUCC 11- } \\
0397\end{array}$ & KR069112 & KR069113 & / & l \\
\hline Arthrinium subroseum & LC7215 & KY494740 & KY494816 & KY705136 & KY705208 \\
\hline Arthrinium subroseum & LC7291 & KY494751 & KY494827 & KY705147 & KY705219 \\
\hline Arthrinium subroseum & LC7292 & KY494752 & KY494828 & KY705148 & KY705220 \\
\hline Arthrinium thailandicum & $\begin{array}{l}\text { MFLUCC 15- } \\
0199\end{array}$ & KU940146 & KU863134 & l & / \\
\hline Arthrinium thailandicum & $\begin{array}{l}\text { MFLUCC 15- } \\
0202\end{array}$ & KU940145 & KU863133 & l & / \\
\hline Arthrinium trachycarpum & CFCC 53038 & МК301098 & / & МК303396 & МК303394 \\
\hline Arthrinium urticae & IMI 326344 & AB220245 & AB220339 & / & AB220292 \\
\hline Arthrinium vietnamensis & IMI 99670 & KX986096 & KX986111 & / & KY019466 \\
\hline Arthrinium xenocordella & CBS 478.86 & KF144925 & KF144970 & KF145055 & KF145013 \\
\hline Arthrinium xenocordella & CBS 595.66 & KF144926 & KF144971 & / & / \\
\hline Arthrinium yunnanum & $\begin{array}{l}\text { MFLUCC 15- } \\
0002\end{array}$ & KU940147 & KU863135 & l & / \\
\hline Arthrinium yunnanum & DDQ00281 & KU940148 & KU863136 & l & l \\
\hline Seiridium phylicae & СРС 19962 & LT853092 & NG_042759 & LT853189 & LT853239 \\
\hline Seiridium phylicae & СРС 19965 & LT853093 & КС005809 & LT853190 & LT853240 \\
\hline
\end{tabular}

Notes: Ex-type strains are in black bold, and newly generated sequences are in blue bold.

Abbreviations: AMH: Ajrekar Mycological herbarium, Pune, Maharashtra, India; CBS: Westerdijk Fungal Biodiversity Institute, Utrecht, Netherlands; CFCC: China Forestry Culture Collection Center, Beijing, China; CGMCC: China General Microbiological Culture Collection Center, Beijing, China; CPC: Culture collection of Pedro Crous, housed at the Westerdijk Fungal Biodiversity Institute; DAOM: Canadian Collection of Fungal Cultures, Ottawa, Canada; DDQ: D.Q. Dai; ICMP: International Collection of Microorganisms from Plants, New Zealand; IFO: Institute for Fermentation, Osaka, Japan; IMI: Culture collection of CABI Europe UK Centre, Egham, UK; JHB: H.B. Jiang; KUMCC: Culture collection of Kunming Institute of Botany, Yunnan, China; LC: Working collection of Lei Cai, housed at CAS, China; MFLUCC: Mae Fah Luang University Culture Collection, Chiang Rai, Thailand; NFCCI: National Fungal Culture Collection of India. 


\section{Results}

\section{Phylogenetic analyses}

Phylogenetic analyses of the combined ITS, LSU, TEF1- $\alpha$ and TUB2 sequence dataset comprise 102 strains. The dataset consists of 3179 total characters including gaps (ITS: 1-750 bp, LSU: 751-1,593 bp, TEF1- $\alpha$ : 1,594-2,217 bp, TUB2: 2,218-3,179). The best scoring ML tree was chosen to represent the phylogenetic relationships of the new taxon with other representative taxa in Arthrinium (Fig. 1), with the final ML optimization likelihood value of -26,209.134905 (ln). All free model parameters were estimated by GAMMA+P-Invar model, with 1,679 distinct alignment patterns and $35.50 \%$ of undetermined characters or gaps. Estimated base frequencies were as follows: $\mathrm{A}=0.236636, \mathrm{C}=0.253087, \mathrm{G}=0.253192, \mathrm{~T}=0.257085$, with substitution rates $\mathrm{AC}=$ 1.324697, $\mathrm{AG}=2.935595, \mathrm{AT}=1.165991, \mathrm{CG}=0.959152, \mathrm{CT}=4.739433, \mathrm{GT}=1.000000$. The proportion of invariable sites $\mathrm{I}=0.316534$, the gamma distribution shape parameter alpha $=$ 0.659233 and the Tree-Length $=4.284453$. In the MP analysis, 1,689 characters were constant, 283 variable characters were parsimony-uninformative, and 1,207 were (included) parsimonyinformative characters. The most parsimonious tree is shown where $\mathrm{TL}=4883, \mathrm{CI}=0.520, \mathrm{RI}=$ $0.832, \mathrm{RC}=0.432, \mathrm{HI}=0.480$. Bayesian posterior probabilities (BYPP) from MCMC were evaluated with a final average standard deviation of split frequencies of 0.009913 .

The phylogenetic tree topology obtained from ML analysis resembled results as previous studies on Arthrinium (Crous \& Groenewald 2013, Dai et al. 2016, 2017, Jiang et al. 2018, Wang et al. 2018, Yan et al. 2019, Pintos et al. 2019, Yang et al. 2019). Phylogenetic trees carried out by ML, MP and BI analyses were similar in overall topologies. Phylogenetic analysis of a combined ITS, LSU, TEF1- $\alpha$ and TUB2 sequence dataset showed that the new taxon clustered within the genus Arthrinium (Apiosporaceae, Xylariales) and has a close relationship with A. garethgonesii (HKAS 96289) with high statistic supported values (100\% MLBP and 100\% MPBP).

\section{Taxonomy}

Arthrinium setostromum H.B. Jiang, K.D. Hyde \& Phookamsak, sp. nov.

Figs 2, 3 Index Fungorum number: IF556844; Facesoffungi number: FoF 06571

Etymology - The specific epithet "setostromum" refers to the ascostromata having black, hair-like setae raising from perithecia through a split of the clypeus.

Holotype: KUN-HKAS 106736

Saprobic on dead bamboo branches. Sexual morph: Ascostromata 250-600 $\mu \mathrm{m}$ long, 140 $180 \mu \mathrm{m}$ high, solitary to gregarious, scattered, immersed in a clypeus, visible as black, raised, lenticular or dome-shaped, becoming superficial on host surface, 1-3-loculate, ostiolate, with a slitlike axis. Ascomata 210-260 $\mu \mathrm{m}$ diam., 100-170 $\mu \mathrm{m}$ high, dark brown to black, perithecial, arranged in a row, clustered, immersed in ascostromata, obpyriform to ampulliform, individually central ostiolate, with pore-like opening, embedded in a clypeus, setose, with dark brown to black, septate, hair-like setae growing from perithecia, raising through a split of the clypeus. Peridium 20$25 \mu \mathrm{m}$ thick, composed several layers of brown to dark brown pseudoparenchymatous cells, paler towards the inner layers, outer layers intermixed with host tissue, arranged in textura angularis to textura prismatica. Hamathecium composed of dense paraphyses, 3-5 $\mu \mathrm{m}$ broad, septate, unbranched, not anastomosing, embedded in gelatinous matrix. Asci 82.5-102.5 $\times 20-30 \mu \mathrm{m}(\bar{x}=$ $92.5 \times 25 \mu \mathrm{m}, \mathrm{n}=20), 8$-spored, unitunicate, broadly cylindrical to clavate, sessile to subsessile, apically rounded, with J- subapical ring. Ascospores $27-33 \times 9.5-12.5 \mu \mathrm{m}(\bar{x}=30 \times 11 \mu \mathrm{m}, \mathrm{n}=$ 20), 2-3-seriate, hyaline, broadly ellipsoidal to clavate, or obovoid, straight to slightly curved near the lower cell, 1-septate, small subglobose at the lower cell and broadly large ellipsoidal at the upper cell, smooth-walled, with multi-guttulate, surrounded by a mucilaginous sheath. Asexual morph: Sporulated on PDA after two months, spore masses visible as dark brown to black on white-grey colonies. Hyphae 1.8-2.5 $\mu \mathrm{m}$ diam., hyaline to pale brown, branched, septate. Conidiophores reduced to conidiogenous cells. Conidiogenous cells 42-66 × 1.5-2.7 $\mu \mathrm{m}(\bar{x}=54 \times$ 
$2.1 \mu \mathrm{m}, \mathrm{n}=20$ ), micronematous, holoblastic, monoblastic, hyaline, cylindrical, flexible to curved, discrete, aseptate, smooth-walled. Conidia 18-20 × 15-19 $\mu \mathrm{m}(\bar{x}=19 \times 17 \mu \mathrm{m}, \mathrm{n}=20)$, acrogenous, brown to dark brown, subglobose to obovoid, 0-1-septate, smooth-walled, multiguttulate, with a truncate basal scar.

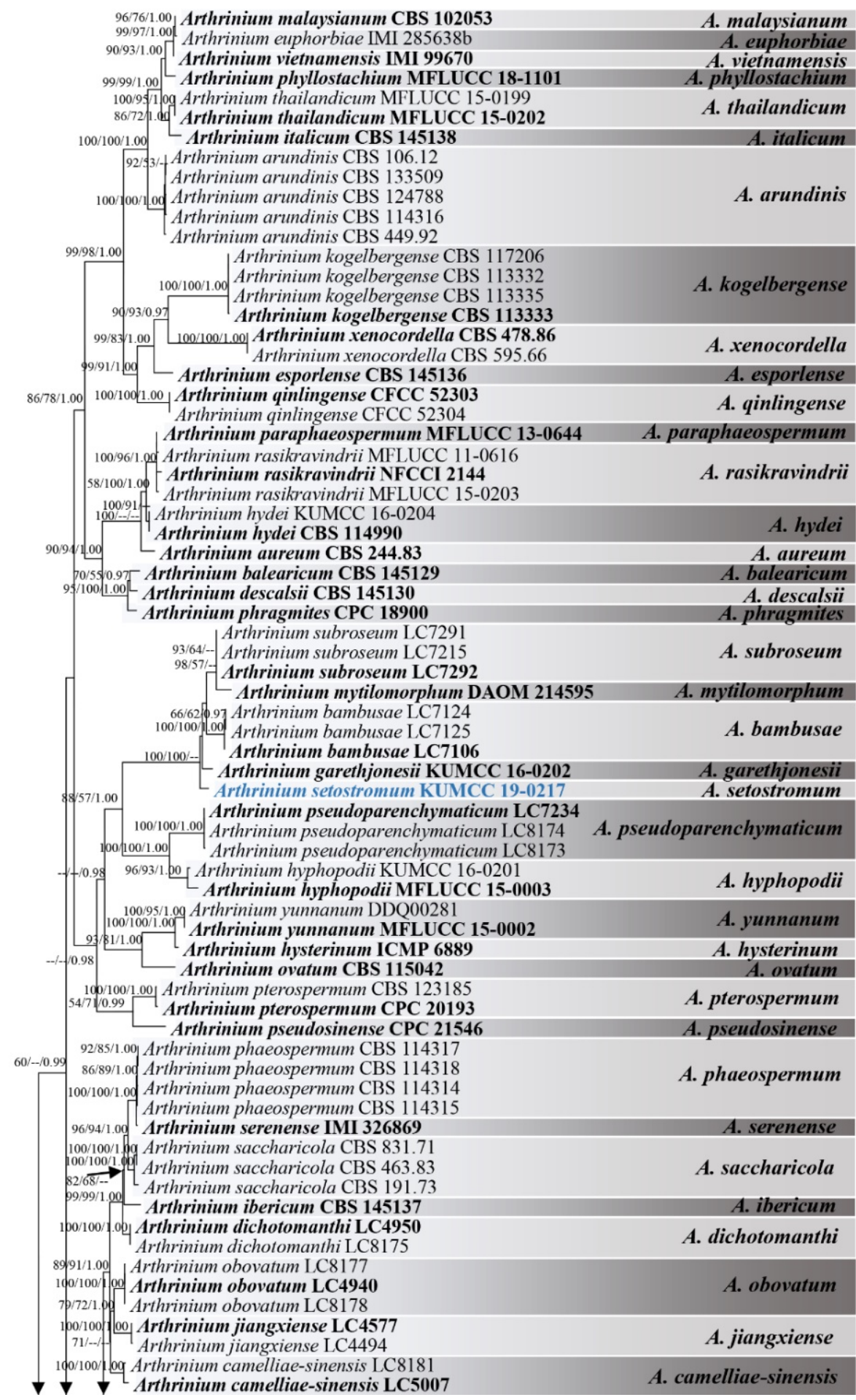

Fig. 1 - Maximum likelihood phylogenetic tree generated by RAxML based on a concatenated ITS, LSU, TEF1- $\alpha$ and TUB2 sequence data. The tree is rooted to Seiridium phylicae (CPC 19962 and CPC 19965). Maximum likelihood (ML), and maximum parsimony (MP) bootstrap values equal to or greater than $50 \%$ and bayesian posterior probabilities (BYPP) equal to or greater than 0.95 are written above the nodes as ML/MP/PP. Ex-type strains are in black bold and newly generated sequence is in blue bold. 


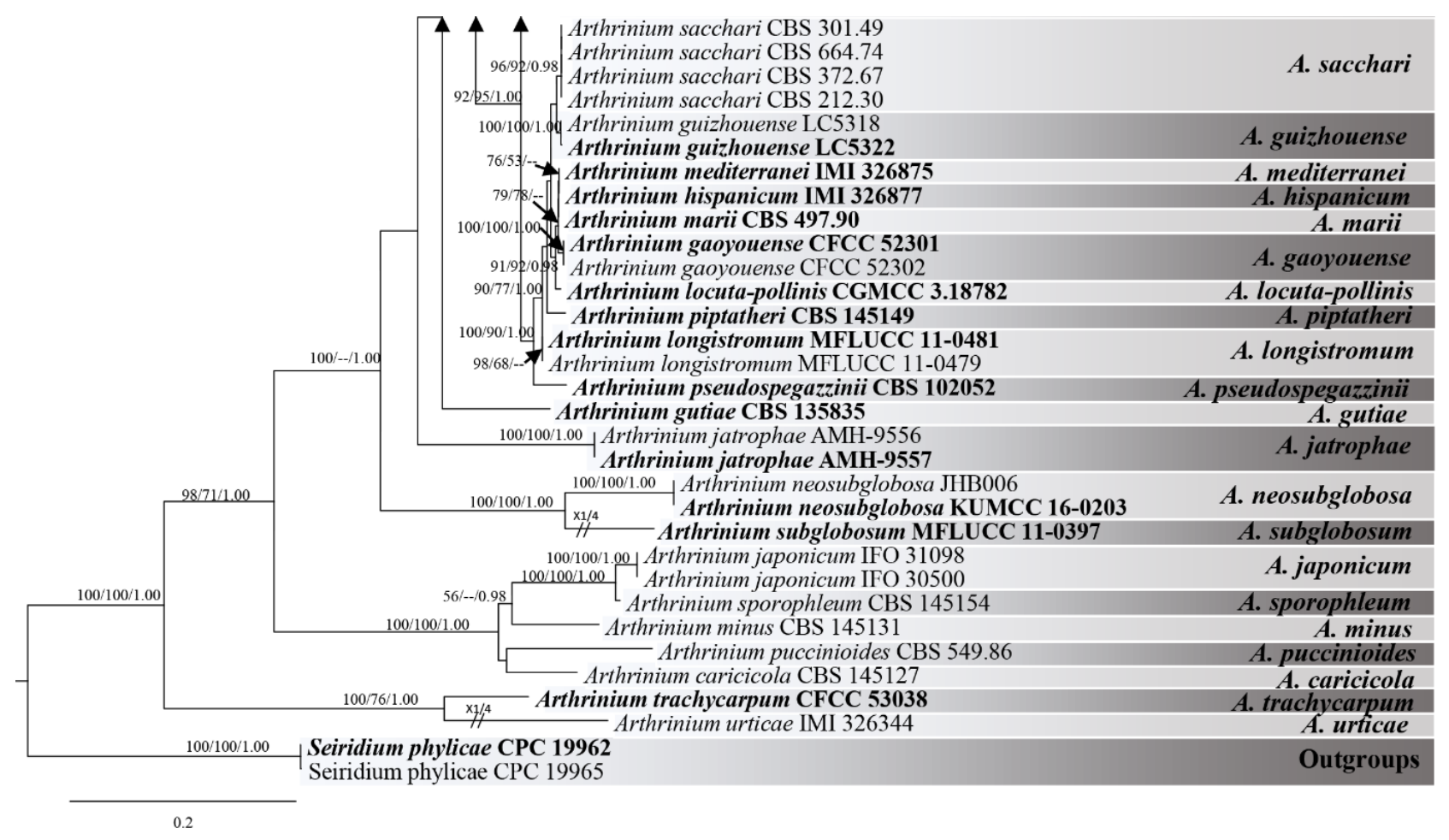

Fig. 1 - Continued.

Culture characteristics - Ascospores germinating on PDA within 24 hrs and germ tubes produced from the upper cell of ascospore. Colonies fast growing on PDA, reaching $35 \mathrm{~mm}$ in 1 week at $20-25^{\circ} \mathrm{C}$, under the normal light, cottony, circular, raised, initially white, becoming greyish, reverse reddish. Mycelium superficial to immersed in media, with branched, septate, smooth hyphae.

Material examined - China, Yunnan Province, Xishuangbanna, Mengla county, Nearby Huiying River, on dead branches of bamboo, 21 January 2019, H.B. Jiang, BN006 (KUN-HKAS 106736, holotype; MFLU 19-2443, isotype), ex-type living cultures, MFLUCC, KUMCC 19-0217.

Notes - Arthrinium setostromum can be distinguished from other phylogenetically related species of Arthrinium in having dark brown to black, hair-like setae growing from perithecia, raising through a clypeus. Morphological comparisons of our novel species with other phylogenetically related taxa are provided in table 2 . Based on a pairwise nucleotide comparison of ITS (Jeewon \& Hyde 2016), A. setostromum differs from A. garethjonesii in 20/ 580 bp (3.45\%) and also differs from A. mytilomorphum in 5/325 bp (1.53\%). Pairwise nucleotide comparison of ITS and TEF1- $\alpha$ also showed that A. setostromum differs from A. bambusae in 24/ 549 bp (4.37\%) and 10/ 313 bp (3.19\%) and differs from A. subroseum in 21/ 548 bp (3.83\%) and 15/ 400 bp (3.75\%).

\section{Discussion}

The genus Arthrinium currently contains 92 epithets in Index Fungorum (2019). However, only 85 described species are presently accepted in Arthrinium, while seven species were transferred to other genera or treated as synonyms of other Arthrinium species (Wang et al. 2017, Index Fungorum 2019, Yang et al. 2019). The phylogenetic placement of 58 Arthrinium species has been confirmed based on molecular data (Wijayawardene et al. 2017, Jiang et al. 2018, Wang et al. 2018, Zhao et al. 2018, Pintos et al. 2019, Yan et al. 2019, Yang et al. 2019).

It is difficult to distinguish most of Arthrinium species based on morphology. Both sexual and asexual morphs of Arthrinium species share similar morphological characteristics, except for some species, such as A. caricicola, A. cuspidatum (Cooke \& Harkn.) Tranzschel and A. luzulae M.B. Ellis. Some Arthrinium species could not be distinguished based on the ITS region, such as $A$. 

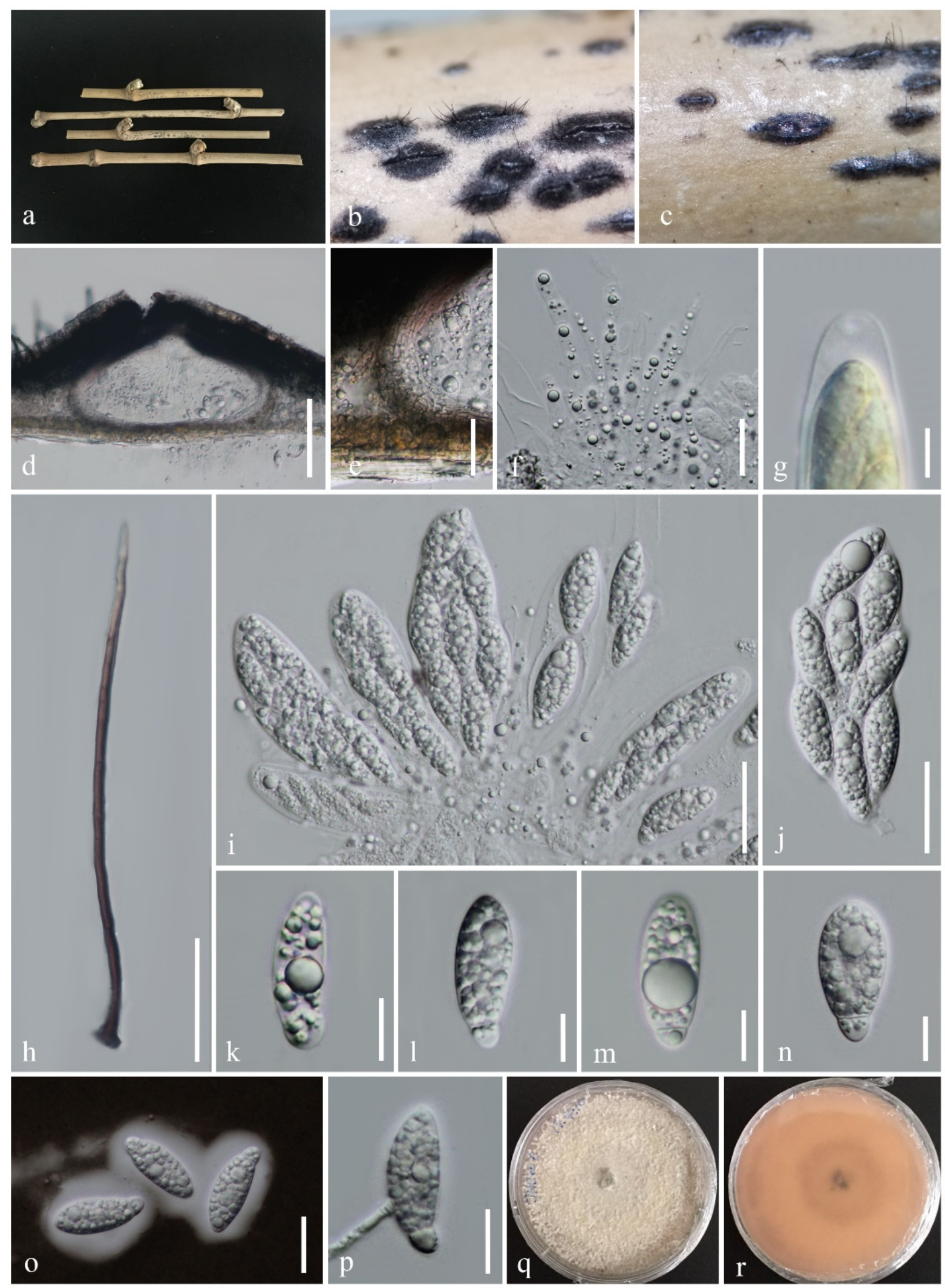

Fig. 2 - Sexual morph of Arthrinium setostromum (KUN-HKAS 106736, holotype). a Dead branches of bamboo. b, c Ascostromata on bamboo host. d Vertical section of ascostroma. e Peridium. f Paraphyses. g J-, Subapical ring stained by Melzer's reagent. h Setae growing from perithecia. i, j Asci. k-n Ascospores. o Ascospores with gelatinous sheath immersing in Indian ink. p Germinating ascospore. $q$, $r$ Culture characteristics on PDA ( $q=$ from above, $r=$ from below). Scale bars: $\mathrm{d}=100 \mu \mathrm{m}, \mathrm{h}=50 \mu \mathrm{m}, \mathrm{e}, \mathrm{i}, \mathrm{j}=30 \mu \mathrm{m}, \mathrm{f}, \mathrm{o}=20 \mu \mathrm{m}, \mathrm{p}=15 \mu \mathrm{m}, \mathrm{k}-\mathrm{n}=10 \mu \mathrm{m}$, $\mathrm{g}=5 \mu \mathrm{m}$. 

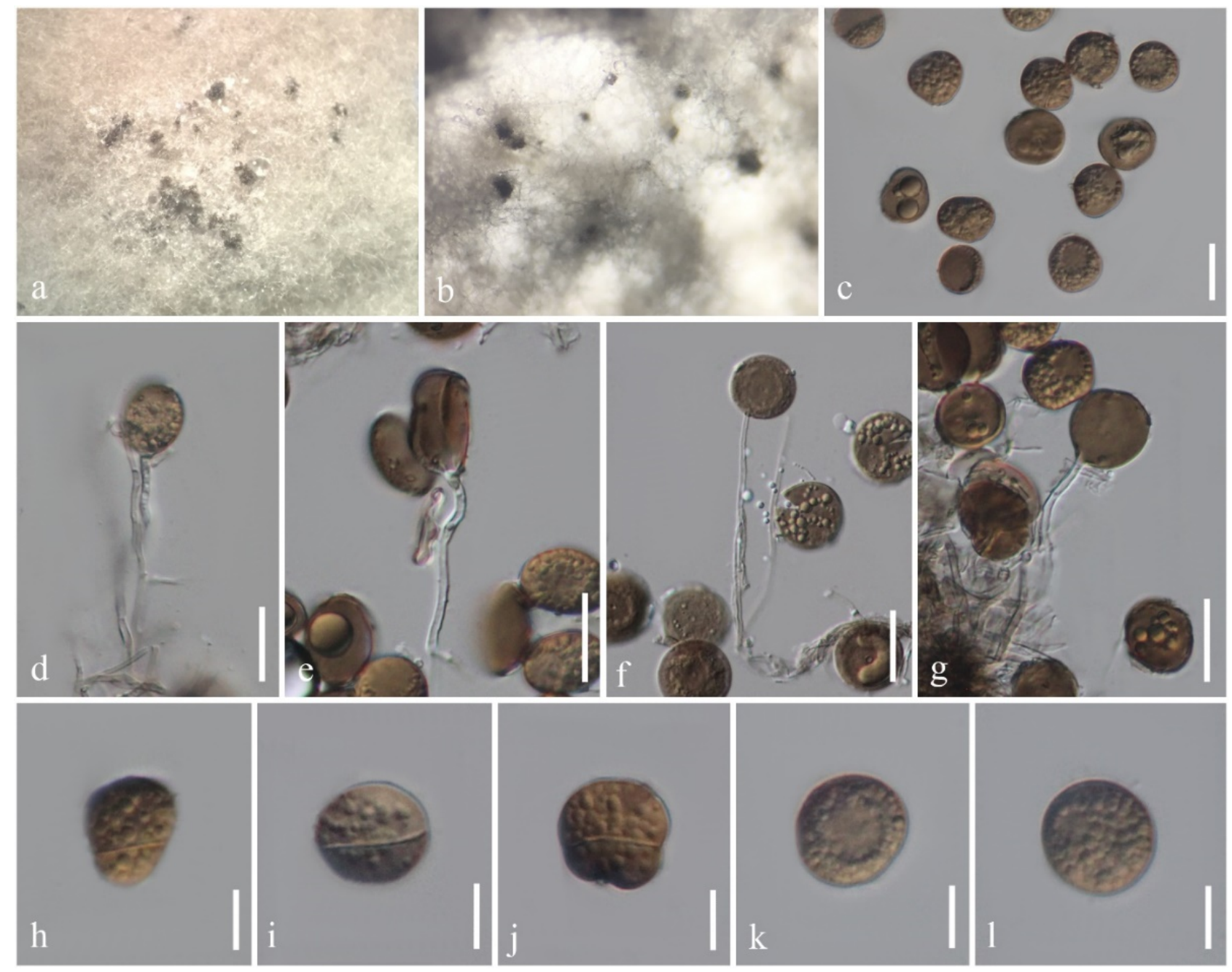

Fig. 3 - Asexual morph of Arthrinium setostromum (from ex-type culture). a, b Colonies on PDA sporulated conidial masses. d-g Conidiogenous cells. c, h-I Conidia. Scale bars: $\mathrm{c}-\mathrm{g}=20 \mu \mathrm{m}$, $\mathrm{h}-\mathrm{I}=10 \mu \mathrm{m}$.

phyllostachium C.L. Yang, X.L. Xu \& K.D. Hyde and A. vietnamensis (Hol.-Jech.) Mei Wang \& L. Cai. Their ITS regions have high similarities (>99\% similarity). However, these species can be distinguished by the protein-coding genes such as TEF1- $\alpha$ and TUB2 (Wang et al. 2018, Yang et al. 2019). Thus, it suggests that multigene phylogenetic analyses based on protein-coding genes coupled with sexual-asexual morphological characteristics are very important to clarify this complex genus Arthrinium.

Arthrinium is widely distributed over the world and lives on various hosts, however, most of them have been found on bamboo plants (Dai et al. 2016, 2017, Jiang et al. 2018, Wang et al. 2018, Farr \& Rossman 2019, Yang et al. 2019). To date, there are 31 recorded species of Arthrinium in China (Hyde et al. 1998, Wang et al. 2018, Zhao et al. 2018, Farr \& Rossman 2019, Yang et al. 2019). Most of the Arthrinium species are distributed in southeast China, south China and southwest China (Farr \& Rossman 2019). Arthrinium qinlingense and A. trachycarpum C.M. Tian \& H. Yan are known from the highest latitude in Shaanxi Province of China (Jiang et al. 2018, Farr \& Rossman 2019, Yan et al. 2019). According to Farr \& Rossman (2019), the specific hosts of Arthrinium species in China are Bambusa and Phyllostachys. 
Table 2 Synopsis of morphological features of the other phylogenetically closely related species to Arthrinium setostromum.

\begin{tabular}{|c|c|c|c|c|c|c|c|c|}
\hline \multirow{2}{*}{ Species name } & \multicolumn{4}{|c|}{ Sexual morph } & \multicolumn{3}{|c|}{ Asexual morph } & \multirow{2}{*}{ References } \\
\hline & $\begin{array}{c}\text { Ascostromata } \\
\text { (long } \times \text { high) }\end{array}$ & $\begin{array}{l}\text { Number } \\
\text { of locules }\end{array}$ & $\begin{array}{l}\text { Asci } \\
(\mu \mathrm{m})\end{array}$ & $\begin{array}{l}\text { Ascospores } \\
(\mu \mathrm{m})\end{array}$ & Conidiophores & $\begin{array}{c}\text { Conidiogenous } \\
\text { cells }\end{array}$ & Conidia & \\
\hline Arthrinium bambusae & N/A & N/A & N/A & N/A & $\begin{array}{l}\text { Reduced to } \\
\text { conidiogenous } \\
\text { cells }\end{array}$ & $\begin{array}{l}\text { 4-12 × 3-7 } \mu \mathrm{m}, \\
\text { aggregated in } \\
\text { clusters on hyphae, } \\
\text { holoblastic, } \\
\text { monoblastic, } \\
\text { hyaline to pale } \\
\text { brown, doliiform to } \\
\text { ampulliform, or } \\
\text { lageniform, smooth }\end{array}$ & $\begin{array}{l}11.5-15.5 \times 7-14 \\
\mu m, \text { olivaceous to } \\
\text { brown, subglobose } \\
\text { to ellipsoid, } \\
\text { aseptate, smooth to } \\
\text { finely roughened }\end{array}$ & $\begin{array}{l}\text { Wang et al. } \\
2018\end{array}$ \\
\hline A. garethjonesii & $\begin{array}{l}1.3-2 \mathrm{~mm} \times \\
188-282 \mu \mathrm{m}\end{array}$ & $3-10$ & $\begin{array}{l}125-154 \times \\
35-42\end{array}$ & $30-42 \times 11-16$ & N/A & N/A & N/A & Dai et al. 2016 \\
\hline A. mytilomorphum & N/A & N/A & N/A & N/A & $\begin{array}{l}\text { Up to } 80 \mu \mathrm{m} \\
\text { long, } 4.5-5 \mu \mathrm{m} \\
\text { wide, arising } \\
\text { from } \\
\text { conidiophore } \\
\text { mother cells }\end{array}$ & $\begin{array}{l}9-10 \times 4.5-5 \mu \mathrm{m}, \\
\text { integrated, terminal } \\
\text { or intercalary, } \\
\text { mono- to } \\
\text { polyblastic, } \\
\text { minutely denticulate }\end{array}$ & $\begin{array}{l}20-30 \times 6-8.5 \mu \mathrm{m} \text {, } \\
\text { dark brown, } \\
\text { fusiform or } \\
\text { navicular, aseptate, } \\
\text { smooth }\end{array}$ & $\begin{array}{l}\text { Bhat \& } \\
\text { Kendrick } 1993\end{array}$ \\
\hline A. setostromum & $\begin{array}{l}250-600 \times \\
140-180 \mu \mathrm{m}\end{array}$ & $1-3$ & $\begin{array}{l}82.5- \\
102.5 \times \\
20-30\end{array}$ & $\begin{array}{l}27-33 \times 9.5- \\
12.5\end{array}$ & $\begin{array}{l}\text { Reduced to } \\
\text { conidiogenous } \\
\text { cells }\end{array}$ & $\begin{array}{l}42-66 \times 1.5-2.7 \\
\mu \mathrm{m}, \text { discrete, } \\
\text { holoblastic, } \\
\text { monoblastic, } \\
\text { hyaline, cylindrical, } \\
\text { flexible to curved, } \\
\text { smooth. }\end{array}$ & $\begin{array}{l}18-20 \times 15-19 \mu \mathrm{m} \text {, } \\
\text { brown to dark } \\
\text { brown, subglobose } \\
\text { to obovoid, } 0-1- \\
\text { sepate, smooth }\end{array}$ & This study \\
\hline A. subroseum & N/A & N/A & N/A & N/A & $\begin{array}{l}\text { Up to } 20 \mu \mathrm{m} \text { long, } \\
2-4.5 \mu \mathrm{m} \text { wide, } \\
\text { sporodochia }\end{array}$ & $\begin{array}{l}3-6.5 \times 2-5 \mu \mathrm{m}, \\
\text { holoblastic, } \\
\text { monoblastic, pale } \\
\text { brown, doliiform to } \\
\text { subcylindrical, } \\
\text { smooth }\end{array}$ & $\begin{array}{l}12-17.5 \times 9-16 \mu \mathrm{m} \text {, } \\
\text { pale brown to dark } \\
\text { brown, globose to } \\
\text { subglobose or } \\
\text { ellipsoidal, aseptate, } \\
\text { smooth }\end{array}$ & $\begin{array}{l}\text { Wang et al. } \\
2018\end{array}$ \\
\hline
\end{tabular}




\section{Acknowledgements}

The authors are grateful to the Mushroom Research Foundation, Chiang Rai, Thailand supporting this research and also acknowledge the Biology Experimental Center, Germplasm Bank of Wild Species, Kunming Institute of Botany, Chinese Academy of Sciences for providing molecular laboratory facilities to extract DNA and amplify PCR fragments. Shaun Pennycook is thanked for his valuable suggestion on naming the new fungus. Rungtiwa Phookamsak thanks CAS President's International Fellowship Initiative (PIFI) for young staff (grant no. Y9215811Q1), the Yunnan Provincial Department of Human Resources and Social Security (grant no. Y836181261), and National Science Foundation of China (NSFC) project code 31850410489 (grant no. Y81I982211) for financial support. Kevin D. Hyde thanks the Thailand Research Fund entitled Impact of climate change on fungal diversity and biogeography in the Greater Mekong Subregion (grant no: RDG6130001), and the Chinese Academy of Sciences, project number 2013T2S0030, for the award of visiting Professorship for Senior International Scientists at Kunming Institute of Botany. Samantha C. Karunarathna thanks CAS President's International Fellowship Initiative (PIFI) for funding his postdoctoral research (number 2018PC0006) and the National Science Foundation of China (NSFC) for funding this work under the project code 31750110478. Jianchu $\mathrm{Xu}$ thanks the Key Research Program of Frontier Sciences of the Chinese Academy of Sciences (grant no. QYZDY-SSW-SMC014). Mingkwan Doilom would like to thank the $5^{\text {th }}$ batch of Postdoctoral Orientation Training Personnel in Yunnan Province and the $64^{\text {th }}$ batch of China Postdoctoral Science Foundation.

\section{References}

Bhat DJ, Kendrick WB. 1993 - Twenty-five new conidial fungi from the Western Ghats and the Andaman Islands (India). Mycotaxon 49, 19-90.

Carbone I, Kohn LM. 1999 - A method for designing primer sets for speciation studies in filamentous ascomycetes. Mycologia 91, 553-556.

Crous PW, Groenewald JZ. 2013 - A phylogenetic re-evaluation of Arthrinium. IMA Fungus 4, 133-154.

Crous PW, Wingfield MJ, Le Roux JJ, Richardson DM et al. 2015 - Fungal planet description sheets: 371-399. Persoonia 35, 264-327.

Dai DQ, Jiang HB, Tang LZ, Bhat DJ. 2016 - Two new species of Arthrinium (Apiosporaceae, Xylariales) associated with bamboo from Yunnan, China. Mycosphere 7, 1332-1345.

Dai DQ, Phookamsak R, Wijayawardene NN, Li WJ et al. 2017 - Bambusicolous fungi. Fungal Diversity 82, 1-105.

Ellis MB. 1971 - Dematiaceous Hyphomycetes. Commonwealth Mycological Institute, Kew, 608 $\mathrm{pp}$.

Farr DF, Rossman AY. 2019 - Fungal Databases, U.S. National Fungus Collections, ARS, USDA. Available from: https://nt.ars-grin.gov/fungaldatabases/ (accessed 17 June 2019).

Felsenstein J. 1985 - Confidence limits on phylogenies: an approach using the bootstrap. Evolution 39, 783-791.

Hall T. 2001 - BioEdit version 5.0.6. North Carolina State University.

Hawksworth DL, Crous PW, Redhead SA, Reynolds DR et al. 2011 - The Amsterdam declaration on fungal nomenclature. IMA Fungus 2, 105-112.

Hyde KD, Fröhlich J, Taylor JE. 1998 - Fungi from palms. XXXVI. Reflections on unitunicate ascomycetes with apiospores. Sydowia 50, 21-80.

Hyde KD, Hongsanan S, Jeewon R, Bhat DJ et al. 2016 - Fungal diversity notes 367-490: taxonomic and phylogenetic contributions to fungal taxa. Fungal Diversity 80, 1-270.

Index Fungorum. 2019 - Available from: https://www.indexfungorum.org/Names/Names.asp. (accessed 25 September 2019).

Jayasiri SC, Hyde KD, Ariyawansa HA, Bhat DJ et al. 2015 - The Faces of Fungi database: fungal names linked with morphology, phylogeny and human impacts. Fungal Diversity 74, 3-18. 
Jeewon R, Hyde KD. 2016 - Establishing species boundaries and new taxa among fungi: recommendations to resolve taxonomic ambiguities. Mycosphere 7, 1669-1677.

Jiang N, Li J, Tian CM. 2018 - Arthrinium species associated with bamboo and reed plants in China. Fungal Systematics and Evolution 2, 1-9.

Katoh K, Rozewicki J, Yamada KD. 2017 - Mafft online service: multiple sequence alignment, interactive sequence choice and visualization. Briefings in Bioinformatics.

Kunze G. 1817 - Zehn neue Pilzgattungen. Mykologische Hefte 1, 1-18.

Maharachchikumbura SSN, Hyde KD, Jones EBG, McKenzie EHC et al. 2016 - Families of Sordariomycetes. Fungal Diversity 79, 1-317.

O’Donnell K, Kistler HC, Cigelnik E, Ploetz RC. 1998 - Multiple evolutionary origins of the fungus causing Panama disease of banana: concordant evidence from nuclear and mitochondrial gene genealogies. Proceedings of the national Academy of Sciences 95, 20442049.

Pintos A, Alvarado P, Planas J, Jarling R. 2019 - Six new species of Arthrinium from Europe and notes about A. caricicola and other species found in Carex spp. hosts. MycoKeys 49, 15-48.

Rambaut A. 2012 - FigTree v. 1.4.0. Available: http://tree.bio.ed.ac.uk/software/figtree/

Rannala B, Yang Z. 1996 - Probability distribution of molecular evolutionary trees: a new method of phylogenetic inference. Journal of Molecular Evolution 43, 304-311.

Ronquist F, Teslenko M, van der Mark P, Ayres DL et al. 2012 - MrBayes 3.2: efficient Bayesian phylogenetic inference and model choice across a large model space. Systematic Biology 61, 539-542.

Seifert K, Morgan-Jones G, Gams W, Kendrick B. 2011 - The Genera of Hyphomycetes. [CBS Biodiversity Series 9]. Utrecht: CBS-KNAW Fungal Biodiversity Centre.

Senanayake IC, Maharachchikumbura SSN, Hyde KD, Bhat JD et al. 2015 - Towards unraveling relationships in Xylariomycetidae (Sordariomycetes). Fungal Diversity 73, 73-144.

Sharma R, Kulkarni G, Sonawane MS, Shouche YS. 2014 - A new endophytic species of Arthrinium (Apiosporaceae) from Jatropha podagrica. Mycoscience 55, 118-123.

Silvestro D, Michalak I. 2011 - raxmlGUI: a graphical front-end for RAxML. Organisms Diversity and Evolution 12, 335-337.

Singh SM, Yadav LS, Singh PN, Hepat R et al. 2012 - Arthrinium rasikravindrii sp. nov. from Svalbard, Norway. Mycotaxon 122, 449-460.

Stamatakis A. 2006 - RAxML-VI-HPC: maximum likelihood-based phylogenetic analyses with thousands of taxa and mixed models. Bioinformatics 22, 2688-2690.

Swofford DL. 2002 - PAUP: phylogenetic analysis using parsimony, version 4.0 b10. Sunderland, MA: Sinauer Associates.

Vilgalys R, Hester M. 1990 - Rapid genetic identification and mapping of enzymatically amplified ribosomal DNA from several Cryptococcus species. Journal of Bacteriology 172, 4238-4246.

Wang M, Liu F, Crous PW, Cai L. 2017 - Phylogenetic reassessment of Nigrospora: ubiquitous endophytes, plant and human pathogens. Persoonia 39, 118-142.

Wang M, Tan XM, Liu F, Cai L. 2018 - Eight new Arthrinium species from China. MycoKeys 34, $1-24$.

White TJ, Bruns T, Lee S, Taylor J. 1990 - Amplification and direct sequencing of fungal ribosomal RNA genes for phylogenetics. Pp 315-322 In: Innis MA, Gelfand DH, Sninsky JJ, White TJ (eds) PCR protocols: a guide to methods and applications. Academic Press, Inc., New York.

Wijayawardene NN, Hyde KD, Rajeshkumar KC, Hawksworth DL et al. 2017 - Notes for genera: Ascomycota. Fungal Diversity 86, 1-594.

Yan H, Jiang N, Liang LY, Yang Q et al. 2019 - Arthrinium trachycarpum sp. nov. from Trachycarpus fortunei in China. Phytotaxa 400, 203-210.

Yang CL, Xu XL, Dong W, Wanasinghe DN et al. 2019 - Introducing Arthrinium phyllostachium sp. nov. (Apiosporaceae, Xylariales) on Phyllostachys heteroclada from Sichuan Province, China. Phytotaxa 406, 91-110. 
Zhao YZ, Zhang ZF, Cai L, Peng WJ et al. 2018 - Four new filamentous fungal species from newly-collected and hive-stored bee pollen. Mycosphere 9, 1089-1116.

Zhaxybayeva O, Gogarten JP. 2002 - Bootstrap, Bayesian probability and maximum likelihood mapping: exploring new tools for comparative genome analyses. Genomics 3, 1-15. 